SHS Web of Conferences 2, 00025 (2012)

DOI: $10.1051 /$ shsconf $/ 20120200025$

(C) Owned by the authors, published by EDP Sciences, 2012

\title{
Nurses' views of infection control organization in regional hospitals of Latvia
}

\author{
D. Platace and I. Millere
}

Rīga Stradiņš University, Latvia

\begin{abstract}
Every nurse can play a significant role in minimizing the risk of healthcare-associated infections, especially, if she use care guidelines and nursing protocols in patients care. The aim of the study to investigate the nurses' views of infection control organization in 10 regional hospitals of Latvia. The qualitative research method as structured interview $(n=36)$ was used. The most common reasons for failures of infection control in nurses' views were referred to the lack of guidelines and patient care documentation, and to the lack of availability of equipment, particularly for hand decontamination, gloveswearing and safe collection of sharp instruments/objects, and to the lack of availability of work wear. The most common reasons why nurses break the rules of the infection control were overload and the lack of knowledge. Conclusions. Obtained results reflect problem areas in patients' care from the nurses' views and necessity for improvement of infection control organization.
\end{abstract}

Key words: infection control, nurses, healthcare-associated infection, infection control organization

The nurse, as the administrator of health care, has an essential role in eliminating the spread of healthcare-associated infections (HAIs) which are related to invasive devices and infection control is the main precondition for qualitative patients care.

However, there exist various factors which may influence it, for instance, infection control organization in the hospital, nursing workload, material-technical supply, guidelines and documentation of the care, as well as nurses' knowledge and attitude (Hugonnet et al., 2007; Jenner et al., 1999).

\section{Materials and methods}

The purpose of the study to establish the nurses' views of infection control organization in high risk units of 10 regional hospitals of Latvia. The qualitative research method as structured interview $(\mathrm{n}=36)$ was used. Structured interview plan was developed, designed and approved by the authors and consisted of 25 questions of infection control activities (safe collection of sharp instruments/objects, work wear providing, precaution measures), infection control organization (nurses' workload, guidelines and patient care documentation, infection control nurses for 250 patients beds) in hospitals and nurses' opinions of infection control efficiency.

\section{Results}

In $1(10 \%)$ of 10 Latvian regional hospitals the number of beds for one nurse's station did not exceed 2 beds in the intensive care unit, but in $9(90 \%)$ hospitals - number of beds for one nurse's station was 3 , in one case even 4 beds in the intensive care unit, while the lowest number of beds stated for the nurse's station in the surgery departments $(13,3 \%)$ were $10-12$ beds. In $9(90 \%)$ of hospitals, starting work in high risk departments, nurses did not have a chance to undergo training on the infection control and in $9(90 \%)$ of regional hospitals a position of infection control nurse unit per 250 patients beds were not take into account as recommendation. In ICUs and in surgery departments of all hospitals there was not

This is an Open Access article distributed under the terms of the Creative Commons Attribution License 2.0, which permits unrestricted use, distribution, and reproduction in any medium, provided the original work is properly cited. 


\section{SHS Web of Conferences}

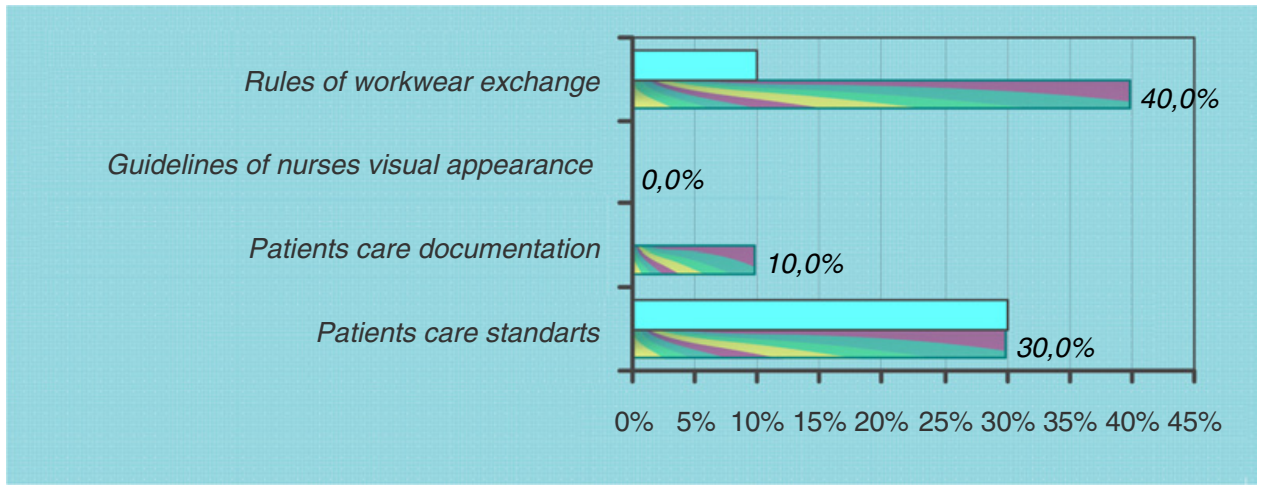

Figure 1. Infection control documentation in patients care of high risk units.

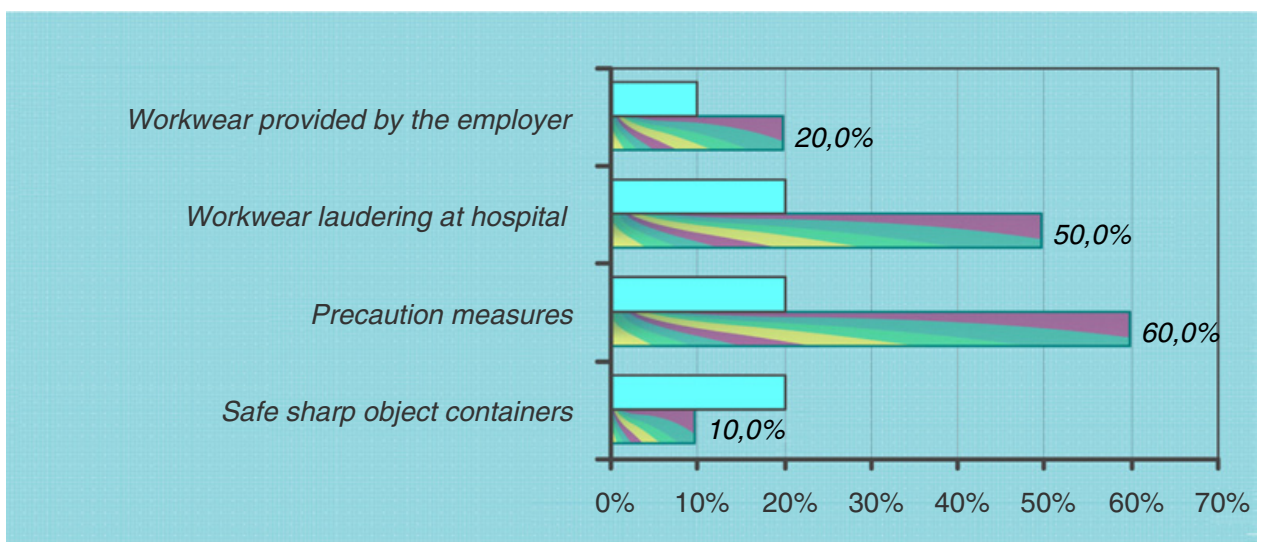

Figure 2. Infection control measures in high risk units of 10 regional hospitals.

established a unified care documentations for patient care with invasive devices, only in one hospital from 10 in the intensive care unit there were care documents and in two hospitals during the research time, patient care documents were being worked out (Figure 1).

In $9(90 \%)$ of hospitals in surgery departments and $8(80 \%)$ of hospitals in ICUs, nurses were not regularly provided with the uniform by the employer. In $8(80 \%)$ of hospitals in surgery departments, nurses were laundering their uniforms at home. Safe sharp object collection containers were not available in $13(87 \%)$ of surgery departments and 9 (90\%) of ICUs (Figure 2), that significantly increased the risk of personnel injury (Stanton et al., 2004).

\section{Discussion}

As shown by several studies, a significant role in limiting the risk of HAIs are care guidelines and nursing protocols for the development and implementation in practice (Gotelli et al., 2008; Morris, Hong Toy, 2008; Saint et al., 2009). Our results showed that a common problem in ICUs and in surgical units was the lack of unified nursing protocols on performing invasive procedures. In the authors' research, according to the respondents' opinion, the most common reasons why nurses break the rules of the infection control are: overload (e.g., "there are cases when a nurse takes care of 40 patients in a ward and 2 MRSA patients", "there are many severely-ill patients, the nurse does not take care of safety but tries to manage the patient care", "most of nurses have more than 1 workload"), are the lack of knowledge 
Int. Conf. SOCIETY. HEALTH. WELFARE; Congr. of Rehabilitation Doctors of Latvia

and understanding on the significance of the infection control (e.g., "they do not see staphylococci, therefore are not scared", "by wearing the gloves, they try to protect themselves and not the patient, because of long nails, rings", " for economic reasons the gloves are disinfected after each patient's care, or they are not worn at all") and the nurses' attitude which might be negligent ("old habits are more comfortable than the new ones", "the attitude depends on the character traits"), as well as insufficient washbasin equipment.

All mentioned, show that Latvian regional hospitals do not comply with all preconditions of the infection control, thus influencing the quality of care, and causing the risk for the development of nosocomial infections, which might be a considerable financial burden for the modest hospital budget.

\section{Conclusions}

The most common reasons for failures of infection control in nurses' views were referred to the lack of guidelines and patient care documentation, and to the lack of availability of equipment, particularly for hand decontamination, gloves-wearing and safe collection of sharp instruments/objects, and to the lack of availability of work wear. According to the respondents' opinion, the most common reasons why nurses break the rules of the infection control were overload of nurses' work and the lack of infection control knowledge. Nurses' believe that there are necessary to invest a lot of energy and resources to improve and optimize infection control organization in regional hospitals of Latvia.

This study was supported by the project of European Social Fond (ESF).

\section{References}

[1] Gotelli, J.M., Merryman, P., Carr, C., McElveen, L., Epperson, C., Bynum, D. (2008) A quality improvement project to reduce the complications associated with indwelling urinary catheters. Urologic Nursing, 28, p. 465-467.

[2] Hugonnet, S., Chevrolet, J.C., Pittet, D. (2007) The effect of workload on infection risk in critically ill patients. Critical Care Medicine, 35, p. 76-81.

[3] Jenner, E.A., Mackintosh, C., Scott, G.M. Infection control- evidence into practice. J of Hospital Infection 1999, 42, p. 91-104.

[4] Morris, W., Hong Toy M. (2008) Strategies for preventing peripheral intravenous cannulae infection. British Journal of Nursing, 10, p. 14-21.

[5] Saint, S., Meddings, J.A., Calfee, D., Kowalski, C.P., Krein, S.L. (2009) Catheter-associated urinary tract infection and the Medicare rule changes. Annals of Internal Medicine, 150, p. $877-884$.

[6] Stanton, M.W., Rutherford, M.K. (2004) Hospital nurse staffing and quality of care. Agency for Healthcare Research and Quality, 14, p. 1-12. 